Recherches en didactique des langues et des cultures

Les cahiers de l'Acedle

13-1 | 2016

Interactions langagières et didactique des langues

\title{
De la pragmatique à la compétence pragmatique
}

A la recherche d'indices dans le Cecrl

\section{Yves Bardière}

\section{(2) OpenEdition}

Journals

Édition électronique

URL : http://journals.openedition.org/rdlc/462

DOI : $10.4000 /$ rdlc.462

ISSN : 1958-5772

Éditeur

ACEDLE

Référence électronique

Yves Bardière, "De la pragmatique à la compétence pragmatique », Recherches en didactique des langues et des cultures [En ligne], 13-1 | 2016, mis en ligne le 25 juillet 2016, consulté le 10 décembre 2020. URL : http://journals.openedition.org/rdlc/462 ; DOI : https://doi.org/10.4000/rdlc.462

Ce document a été généré automatiquement le 10 décembre 2020.

\section{(c)}

Recherches en didactique des langues et des cultures is licensed under a Creative Commons AttributionNonCommercial-NoDerivatives 4.0 International License 


\title{
De la pragmatique à la compétence pragmatique
}

\author{
A la recherche d'indices dans le Cecrl
}

Yves Bardière

\section{Introduction}

1 Les théories de référence fournissent aux chercheurs didacticiens l'« ancrage théorique " nécessaire à l'élaboration des différentes méthodologies. Celles-ci inspirent la didactique institutionnelle déclinée notamment dans les instructions officielles, ce dernier niveau correspondant à l'« ancrage officiel ». Il se dessine ainsi ce que C. Bourguignon nomme la « chaîne didactique » (Bourguignon, 2005 :12).

Figure 1 : La chaîne didactique

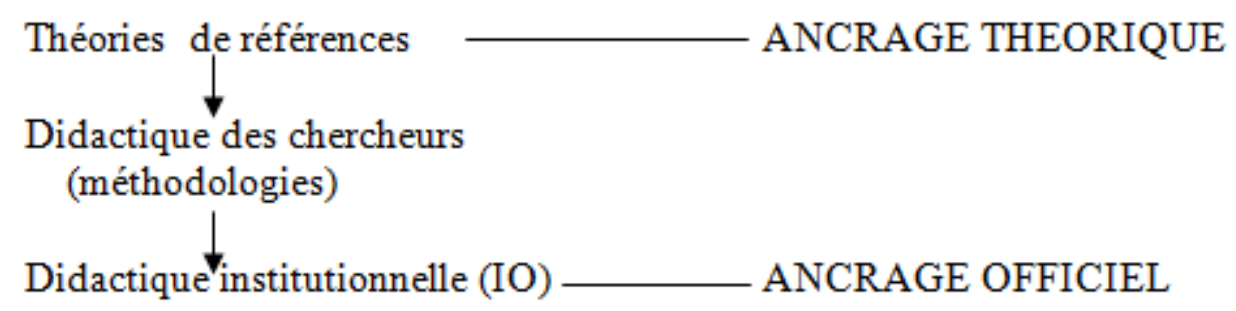

2 L'étude proposée ici s'intéresse à l'un des courants de recherche actuellement les plus vivants en sciences du langage, la pragmatique et ses applications didactiques. Elle vise à mieux cerner le passage de l'ancrage théorique à l'ancrage officiel, c'est-à-dire la manière dont la didactique institutionnelle contemporaine, telle qu'elle apparaît notamment dans le cadre européen, s'empare de la pragmatique en tant que théorie de référence. Il s'agit en d'autres termes d'évaluer la nature des emprunts notionnels prélevés par le Cecrl et les textes officiels sur une discipline scientifique.

On soulignera donc d'ores et déjà la distinction lexicale entre la science et son objet tout en tissant des liens entre les deux. Le terme «pragmatique » apparait généralement sous 
sa forme nominale dans le premier cas, sous sa forme adjectivale dans le second. La consultation des dictionnaires de sciences du langage, tels que le Nouveau dictionnaire encyclopédique des sciences du langage de Ducrot et Schaeffer (1972), le Sémiotique dictionnaire raisonné de la théorie du langage de Greimas \& Courtès (1979) et le plus récent Dictionnaire d'Analyse du discours de Charaudeau \& Maingueneau (2002) montre en effet que, sur 40 ans environ, le terme a toujours uniquement désigné la discipline de recherche et non son objet. Pour référer à celui-ci, les textes officiels qui régissent l'enseignement des langues vivantes recourent à l'adjectif : le Cecrl parle de «compétence pragmatique», d'«aptitude pragmatique», de "composante pragmatique», de "fonction pragmatique ", d' "erreur pragmatique ", de " gains pragmatiques », etc.

Force est de constater que le terme de "pragmatique», qu'il s'agisse du nom ou de l'adjectif, suscite un certain flou conceptuel chez les futurs enseignants du primaire et du secondaire, voire les enseignants déjà titulaires. Cela s'explique peut-être non seulement par une confusion fréquente entre la science et son objet mais également par le fait que la pragmatique, en tant que théorie de référence, s'est régulièrement trouvée enrichie, au fil des ans, d'acceptions nouvelles, plus ou moins complexes, visant à compléter, parfois à se substituer aux analyses précédentes: pragmatique indexicale mise en évidence par Morris (1939) et développée par Jakobson (1963), pragmatique sémantique fondée sur la théorie des actes de langage d'Austin (1962) puis de Searle (1972), pragmatique radicale étudiée, entre autres, par Grice (1975) et Sperber \& Wilson (1986), pragmatique fictionnelle initiée par Searle puis affinée par Schaeffer (1999), à travers, notamment, le concept de contrat pragmatique, pragmatique interactionnelle appréhendée par Roulet \& al. (1985), Burger (2001), Fillietaz (2001), etc. Il n'est guère étonnant que dans ce dédale notionnel l'enseignant finisse par s'égarer, d'autant plus que le Cecrl, texte de référence pour l'enseignement des langues vivantes, propose une approche définitoire de la composante pragmatique qui, à première vue, ne présente que des relations plus ou moins distendues avec les théories de références élaborées par la plupart des linguistes et philosophes.

Pour mieux cerner le rôle que joue la compétence pragmatique dans le dispositif didactique, je rappellerai brièvement les principales acceptions dont la pragmatique, en tant que domaine de recherche, s'est trouvée régulièrement investie. C'est sous cet éclairage diachronique et scientifique que j'examinerai plus en détail l'approche définitoire du Cadre européen, sur lequel se fondent les Instructions officielles pour l'enseignement des langues vivantes.

\section{L'ancrage théorique}

6 Très schématiquement, la pragmatique concerne l'étude de l'usage du langage. Elle s'intéresse à l'énoncé. L'énoncé est la phrase enrichie des données contextuelles dans laquelle elle est produite, la phrase complétée par les informations fournies par la situation. C'est la raison pour laquelle la pragmatique est avant tout considérée comme une théorie de la communication linguistique. Dans une situation de communication, les locuteurs n'échangent pas des phrases, ils échangent des énoncés. Les choses se compliquent dès lors que l'on tente une approche définitoire plus précise. 


\section{La pragmatique intégrée à la sémantique}

\section{De la situation à la parole : la pragmatique indexicale}

7 Les premières avancées scientifiques présentent la pragmatique comme faisant partie intégrante de la linguistique, d'où les expressions de "pragmatique intégrée ", " pragmatique intégrée à la sémantique » ou encore " pragmatique linguistique » parfois utilisées pour référer à cette discipline. Dans le Nouveau dictionnaire encyclopédique des sciences du langage, Ducrot et Schaeffer (1972:131-133) proposent la double acception suivante :

1. La pragmatique étudie tout ce qui, dans le sens d'un énoncé, tient à la situation dans laquelle l'énoncé est employé, et non à la seule structure linguistique de la phrase utilisée. [...]

2. La pragmatique concerne non pas l'effet de la situation sur la parole, mais celui de la parole sur la situation.

8 La première définition souligne l'apport exophorique dans l'intelligibilité du message ou, dit autrement, l'éclairage du message linguistique par la situation d'énonciation. C'est le philosophe américain Morris ${ }^{1}$ (1938) qui, en 1938, a mis en évidence les premiers traits distinctifs de ce concept. Pour Morris, la pragmatique était principalement restreinte à l'étude des pronoms de première et deuxième personne et à celle des adverbes de temps et de lieu (ici, maintenant), mots qui ont pour propriété commune de tirer leur signification de données partiellement extérieures au langage lui-même, c'est-à-dire de la situation dans laquelle la communication se produit.

9 Jakobson (1963) a par la suite dénommé ces « universaux » de langage, « embrayeurs » (en anglais shifters), terme auquel on préfère souvent aujourd'hui une appellation empruntée à Peirce, celle de "déictiques». Les déictiques sont ces mots qui s'indexent ${ }^{2}$ sur la situation d'énonciation, d'où le nom de " pragmatique indexicale ", parfois donnée à cette approche. Les démonstratifs, qui impliquent une monstration, mais aussi, nous l'avons vu, les pronoms personnels de l'interlocution, sont à ranger parmi les déictiques. De même, les temps verbaux désignant un événement antérieur ou postérieur au moment de l'énonciation $(T o)$ sont rattachés à cette catégorie.

\section{De la parole à la situation : la pragmatique fondée sur la théorie des actes de langage}

10 La deuxième définition «inverse " le point de vue de la première, sous la forme d'une antimétabole : «la pragmatique concerne non pas l'effet de la situation sur la parole, mais celui de la parole sur la situation » (1972: 133). Cette deuxième acception de la pragmatique est issue des analyses du philosophe anglais, Austin, qui considère «l'acte de langage (parfois dénommé l'acte de parole ou l'acte de discours) [comme] une des notions essentielles de la pragmatique linguistique » (cité par Maingueneau, 1996b: 10). Au terme de son parcours réflexif, Austin distingue trois composantes indissociables de tout acte de langage, ces trois composantes pouvant être simultanées. La première composante est locutoire ou locutionnaire : elle concerne l'articulation et la combinaison des sons ainsi que le choix des mots, les combinaisons syntaxiques des notions représentées par les mots, l'agencement cohérent des phrases, etc. Cette dimension sera nettement privilégiée par le Cecrl dans son approche de la compétence pragmatique. Sans elle, il n'y aurait aucune mise en œuvre du langage. La seconde composante est illocutoire ou illocutionnaire : elle vise à agir directement sur la situation, à transformer les rapports entre interlocuteurs. 
La signification qu'Austin mettait initialement derrière "performatif» se retrouve largement dans la notion d'acte illocutionnaire. La troisième composante est perlocutoire ou perlocutionnaire. L'énonciation sert des fins plus lointaines. La perlocution agit indirectement sur la situation, dont elle dépend entièrement. Elle consiste à produire des effets ou conséquences sur les interlocuteurs (comme un mouvement, la peur, le rire ou le chagrin). L'acte perlocutoire décrit donc les conséquences qu'un acte illocutoire peut avoir. Par exemple, " promettre », « obliger », « affirmer » sont des actes illocutoires, codés linguistiquement, mais le fait qu'une promesse puisse faire plaisir, qu'un ordre puisse embarrasser, qu'une assertion puisse surprendre, relève de la perlocution.

\section{La pragmatique radicale}

11 À la pragmatique intégrée, s'oppose une autre approche, plus novatrice, la « pragmatique radicale ", à laquelle on associe notamment les noms de Grice, Gazdar, Levinson et Sperber \& Wilson. Ces théories introduisent une séparation entre la linguistique et la pragmatique. La pragmatique radicale devient donc une discipline à part entière, un domaine autonome et séparé de la sémantique. Elle prend en charge tout ce qui, dans l'interprétation de l'énoncé, ne se fait pas de façon codique. Elle postule que la communication verbale est le plus souvent non-littérale ou implicite. Ce sont donc aux inférences pragmatiques, qui viennent compléter le message codique pour livrer une interprétation complète des énoncés, que s'intéresse cette nouvelle approche.

Voici à titre d'illustration un exemple emprunté à Moeschler (2001:5) dans Pragmatique: état et perspectives:

Contexte : Il est huit heures du soir, heure à laquelle les enfants de la famille $\mathrm{M}$ se couchent.

Le père à son fils Axel : «Va te laver les dents.

Axel à son père : «Je n'ai pas sommeil ».

13 En disant « Va te laver les dents », le père implicite " va te coucher ». En répondant " Je n'ai pas sommeil », le fils signifie qu'il ne veut pas aller se laver les dents maintenant et donc aller se coucher maintenant. Il y a, on le voit, une distance considérable entre ce que le père et le fils disent et ce qu'ils communiquent.

14 C'est par le processus inférentiel que l'auditeur ou le lecteur fait des hypothèses sur le vouloir-dire du locuteur ou du scripteur et réussit à saisir son intention de communication. Il ressort clairement de cet exemple que la communication se fonde sur la présomption que les interlocuteurs coopèrent dans la communication. Cette approche inférentielle relève donc d'un modèle pragmatique cognitif de la communication. Elle s'est érigée en principe dans la théorie de la pertinence, qui a été élaborée à partir des maximes conversationnelles de Grice (1975) (partiellement reprises dans le Cecrl) et qui stipule que tout acte de communication ostensive-inférentielle "communique une présomption de sa propre pertinence optimale » (Moeschler \& Reboul, 1994).

Un acte de communication ostensive-inférentielle indique qu'il est un acte de communication et qu'il requiert un processus inférentiel pour l'interpréter. Par exemple, une personne qui, dans la rue, tire quelqu'un par la manche pour l'avertir d'un danger quelconque, accomplit un acte de communication ostensive-inférentielle. Cette personne agit en effet de manière ostensive en mobilisant l'attention de l'autre : elle le tire par la manche (intention communicative) et elle lui montre le danger (intention informative). L'individu ainsi alerté tente alors spontanément de comprendre l'intention 
communicative et informative de l'autre (dimension inférentielle). La communication ostensive-inférentielle ne porte pas uniquement sur la communication linguistique mais sur la communication en général, verbale ou non verbale.

\section{L'organisation du discours}

16 Il existe enfin d'autres théories plus controversées quant à la délimitation de leur champ d'étude et - ceci expliquant cela - leur appartenance à la pragmatique, comme la linguistique textuelle, "quelquefois considérée comme une partie de la pragmatique 》 (Ducrot \& Schaeffer, 1992) ou l'analyse du discours. La linguistique textuelle couvre en effet un domaine aux frontières diversement définies et elle est souvent confondue avec l'analyse du discours, qui se prête, elle aussi, à des définitions plus ou moins larges. Comme le souligne Maingueneau (1996a : 83) :

Certains lui donnent avant tout pour objet les phénomènes de cohésion, d'autres, en s'orientant vers les problèmes de genre et de typologie du discours, sont très proches des perspectives de l'analyse du discours, la délimitation respective de ces deux domaines étant sujette à controverses.

\section{La linguistique textuelle}

Pour maintenir la distinction avec l'analyse du discours, je considérerai que la linguistique textuelle traite fondamentalement des phénomènes de cohésion et de cohérence. Ces derniers jouent, nous le verrons, un rôle essentiel dans la compétence pragmatique discursive telle que la décrit le Cecrl.

Il convient de souligner ici l'influence déterminante de Halliday et Hasan (1976) dans la mise en place et la diffusion de ces notions. Les auteurs établissent une distinction entre les plans intra-phrastique (cohésion interne) et extra-phrastique (cohésion externe). Dans le premier cas, «toutes les unités grammaticales - les phrases, les syntagmes, les groupes de mots et les mots - sont cohésives de manière interne simplement parce qu'elles sont structurées » (1976: 7). Au-delà de la phrase, la cohésion devient externe. Elle s'exprime à l'aide d'items lexico-grammaticaux qui tissent des liens sémantiques entre les phrases. Ces relations de cohésion externe ne confèrent pas au texte sa structure mais contribuent simplement à sa texture. Qu'elles relèvent du niveau interne ou externe, ces marques de relations exercent une fonction similaire. C'est la raison pour laquelle « depuis M.A.K. Halliday et $R$. Hasan (1976), on tend à les regrouper sous le nom générique de cohésion » (Charolles, 1988 : 53).

19 A cette cohésion fondamentalement sémantique, le fondateur de la linguistique systémique fonctionnelle ajoute une dimension sociale : "la langue fait partie du système social» (Halliday, 1978: 39). Cette approche se trouve régulièrement réaffirmée, notamment dans An Introduction to Functional Grammar (1985) et Language and Society (2009). Les composantes linguistique et sociale exercent une influence mutuellement réciproque et potentiellement dynamique: la situation sociale génère le discours, susceptible de modifier la situation, laquelle peut à son tour changer le discours. L'adéquation entre discours et situation est source de cohérence ( $c$ f. infra l'analyse du discours).

Si la démarcation entre cohésion et cohérence est parfois délicate à établir, elle n'en demeure pas moins fondamentale, un texte pouvant être cohésif sans être pour autant cohérent. Si je dis: «j'ai lu les premières pages du Cecrl; je me suis endormi », j'obtiens une suite à la fois cohésive et cohérente. En revanche, «j'ai lu les premières pages du Cecrl; j’ai 
assommé mon voisin ", est une suite cohésive mais à première vue incohérente, sauf à considérer que le voisin en question sert de défouloir et permet ainsi à l'auteur du discours d'évacuer l'ennui et l'irritation générés par la lecture d'un texte jugé soporifique.

La cohésion d'un texte concerne donc l'enchaînement discursif des unités qui le composent. Elle sollicite des paramètres linguistiques tels la phoricité (endophorique mais aussi exophorique), la présence de connecteurs ou de liens logiques implicites, une progression thématique générée entre autres par le passage régulier du statut de rhème à celui de thème, etc. Ces phénomènes linguistiques sont le ciment cohésif qui soude des unités linguistiques sinon simplement juxtaposées. Ils assurent la continuité du texte et le font avancer. La cohérence est, quant à elle, davantage liée au contexte, au type de situation dans laquelle se trouvent les interlocuteurs, à leur connaissance partagée du monde extralinguistique. Un texte doit être en effet en adéquation avec son contexte d'utilisation (cf. supra le lien entre langue et environnement social). Par exemple, un même énoncé pourra sembler cohérent ou incohérent selon qu'il est prononcé lors d'un colloque de linguistique, d'une excursion en montagne ou d'un exercice opérationnel multinational en Adriatique. La cohérence concerne le lien entre texte et contexte, entre parole et situation et relève donc bien in fine de la pragmatique (cf Maingueneau, 1996b : 16).

Le rapport de cause à effet entre cohésion et cohérence (la cohésion est-elle un facteur de cohérence ?) et inversement entre cohérence et cohésion (la cohérence est-elle un facteur de cohésion ?) a suscité de nombreuses remarques. Par exemple, pour Halliday et Hasan (1976), la cohésion n'est pas suffisante mais elle est nécessaire à la cohérence. Pour Charolles (1988) ou Moeschler \& Reboul (1994), elle n'est ni suffisante ni nécessaire. Dans Cohésion-cohérence: accords et désaccords (2006), Mathilde Salles propose une synthèse claire et argumentée sur le sujet.

\section{L'analyse du discours}

Elle fait l'objet d'acceptions larges et variées, mais pour limiter son extension, j'adopterai ici la définition mise en avant par Maingueneau (1996b : 11 ; souligné dans le texte) :

Nous jugeons préférable de spécifier l'analyse du discours comme la discipline qui, au lieu de procéder à une analyse linguistique du texte en lui-même ou à une analyse sociologique ou psychologique de son "contexte", vise à articuler son énonciation sur un certain lieu social. Elle a ainsi affaire aux genres de discours à l'œuvre dans les secteurs de l'espace social (un café, une école, une boutique...), ou dans les champs discursifs (politique, scientifique...).

Fillietaz, Roulet \& Grobet (2001) proposent un modèle d'analyse du discours qui procède de cette définition, et sur lequel je m'appuierai pour étudier le texte européen. Ce modèle se fonde sur la mise en évidence des principes et régularités qui président à la construction et l'interprétation des textes et des dialogues et vise ultimement à dresser une typologie des discours. Fillietaz (2001: $102 \mathrm{sq}$ ), notamment, établit une distinction pertinente entre "représentation schématique», praxéologique (cf. infra $\S$ 2.3.2.) et conceptuelle et "configuration émergente ", également praxéologique et conceptuelle. La première renvoie à un schéma typifiant, pré-existant, régulé par le savoir partagé des interlocuteurs, la seconde à des situations d'interaction effectives, liées à une réalité discursive spécifique et faisant donc l'objet de négociations situées. Toute représentation 
schématique repose sur le principe de typicalité élaboré à partir d'expériences antérieures. Comme le fait remarquer l'auteur :

Les événements passés laissent des traces dans l'expérience du présent, si bien que les rapports des individus à leur environnement ne sont pas radicalement inventés par eux, mais nécessairement médiatisés par des indexations sociales et des savoirfaire collectifs intériorisés (op.cit : 105).

Cette approche revêt un grand intérêt pour mieux cerner les enjeux de la composante pragmatique fonctionnelle telle qu'elle se trouve exposée dans le Cecrl.

\section{L'ancrage officiel : le Cecrl} développées par la recherche scientifique. Il se dit clairement «non dogmatique » : «il n'est rattaché de manière irrévocable et exclusive à aucune des théories ou pratiques concurrentes de la linguistique ou des sciences de l'éducation " (CECR : 13; souligné dans le texte). Cette prise de position explique, dans une large mesure, la distance plus ou moins marquée qu'opère le Cecrl avec les différentes acceptions de la pragmatique retenues dans le domaine scientifique. La transposition didactique ${ }^{3}$ pratiquée par les auteurs du texte européen vise à construire un référent commun orienté non pas vers l'acquisition de cadres théoriques universitaires mais vers la construction d'un référent théorique professionnel partagé par tous les usagers. Le Cecrl s'adresse donc à des utilisateurs qui ne sont pas nécessairement des spécialistes du domaine de recherche sous-jacent.

$\mathrm{Au}$ sein de la compétence pragmatique, le cadre européen opère une distinction entre compétences « discursive », «fonctionnelle » et de « conception schématique »:

La compétence pragmatique traite de la connaissance que l'utilisateur/apprenant a

des principes selon lesquels les messages sont:

a. organisés, structurés et adaptés (compétence discursive)

b. utilisés pour la réalisation de fonctions communicatives (compétence fonctionnelle)

c. segmentés selon des schémas interactionnels et transactionnels (compétence de conception schématique) (Cecrl : 96).

Seules les compétences «discursive » et "fonctionnelle» donnent lieu à des descriptifs détaillés, rien n'est dit en revanche de la compétence de « conception schématique », sauf à considérer que celle-ci ait été intégrée dans la compétence fonctionnelle, ce qui, sauf erreur d'interprétation, me paraît être le cas.

\section{La compétence discursive ${ }^{4}$}

\section{L'héritage des théories scientifiques}

\section{La linguistique textuelle}

Pour illustrer la compétence pragmatique discursive, le texte européen met fondamentalement l'accent sur l'organisation cohérente et cohésive du discours. Les termes et expressions « organisation des phrases 》, " organisation thématique », « organisation logique ", «structuration », « cohérence ", « cohésion ", « enchaînement naturel », etc. saturent les quelques pages (CECR : 96-98) dévolues à cette présentation. Le texte ne s'appuie donc pas sur les théories scientifiques les plus communément mises en avant pour définir la pragmatique. Il s'intéresse fondamentalement à l'aspect locutoire (v. supra § 1.1.2.) de la 
langue et se fonde pour l'essentiel sur une approche de la pragmatique qui relève de la linguistique textuelle. L'étude de la cohérence et de la cohésion d'un texte est en effet l'objet de la linguistique textuelle (v. supra § 1.3.).

Il me paraît possible de hiérarchiser cohérence et cohésion car, comme le fait remarquer Maingueneau, "la cohérence s'appuie sur la cohésion» (1996b: 16). Autrement dit, la cohérence apparaît comme la condition sine qua non de l'intelligibilité du message. Or le texte européen regroupe ces deux notions sans véritablement les distinguer, mais surtout il les met sur le même plan que les autres items répertoriés, sans prendre en compte les relations d'ordre hypero-hyponymique qui s'établissent pourtant entre eux. En l'occurrence, l'item " cohérence et cohésion » placé sous l'en-tête « la capacité de gérer et de structurer le discours » est suffisamment extensif pour pouvoir servir lui-même de titre à l'ensemble de cette partie.

\section{Les maximes conversationnelles de Grice}

La seule référence explicite à l'héritage intellectuel issu des grandes théories élaborées par la recherche sur la pragmatique consiste en un rappel des maximes conversationnelles de Grice. Voici le passage concerné :

La compétence discursive [...] recouvre :

- la capacité de gérer et de structurer le discours en termes :

- d'organisation thématique

- de cohérence et de cohésion

- d'organisation logique

- de style et de registre

- d'efficacité rhétorique

- de principe coopératif

Faites en sorte que votre contribution corresponde à ce qui est exigé, au niveau où elle a lieu, par la finalité ou le sens acceptés de l'échange conversationnel dans lequel vous êtes engagé(s), en observant les principes suivants :

- la qualité (essayez de rendre votre contribution véridique);

- la quantité (rendez votre contribution aussi informative que possible mais pas plus) ;

- la pertinence (ne dites que ce qui est approprié) ;

- la modalité (soyez bref et précis ; évitez l'obscurité et l'ambiguïté). (Grice, 1975 :

96)

Les neuf maximes de Grice ont été regroupées en quatre catégories universelles empruntées à Kant et c'est sous cette forme qu'elles apparaissent, à quelques détails près, dans le Cecrl, sous l'item "principe coopératif " (maximes conversationnelles de Grice, 1975), au § 5.2.3. Les évolutions post-gricéennes, qui se caractérisent par une réduction successive du système à deux puis à un seul principe, le principe de la pertinence, ne sont pas prises en compte dans le Cadre Européen. Le tableau 1 résume les maximes conversationnelles de Grice reprises dans le Cecrl :

Tableau 1 : Les maximes conversationnelles de Grice prélevées par le Cecrl

\begin{tabular}{|l|l|l|}
\hline GRICE & $\begin{array}{l}\text { QUATRE } \\
\text { UNIVERSELLES }\end{array}$ & CATEGORIES \\
CECRL \\
\hline
\end{tabular}




\begin{tabular}{|c|c|c|}
\hline $\begin{array}{l}\text { 1) Donnez autant } \\
\text { d'information qu'il est } \\
\text { requis. }\end{array}$ & \multirow{2}{*}{$\begin{array}{l}\text { - Deux maximes de quantité }: 1 \text { ) } \\
\text { et } 2 \text { ) }\end{array}$} & \multirow{2}{*}{$\begin{array}{l}\text { - La quantité (rendez votre } \\
\text { contribution aussi informative } \\
\text { que possible mais pas plus) }\end{array}$} \\
\hline $\begin{array}{l}\text { 2) } \mathrm{Ne} \text { donnez pas plus } \\
\text { d'information qu'il n'est } \\
\text { requis. }\end{array}$ & & \\
\hline $\begin{array}{l}\text { 3) N'affirmez pas ce pour } \\
\text { quoi vous manquez de } \\
\text { preuves. }\end{array}$ & \multirow{2}{*}{$\begin{array}{l}\text { - Une maxime de qualité } \\
\text { déclinée en deux sous- } \\
\text { maximes : 3) et } 4 \text { ) }\end{array}$} & \multirow{2}{*}{$\begin{array}{l}\text { - La qualité (essayez de rendre } \\
\text { votre contribution véridique) }\end{array}$} \\
\hline $\begin{array}{l}\text { 4) N'affirmez pas ce que } \\
\text { vous croyez faux. }\end{array}$ & & \\
\hline 5) Parlez à propos. & $\begin{array}{l}\text { - Une maxime de relation ou de } \\
\text { pertinence }\end{array}$ & $\begin{array}{l}\text { - La pertinence (ne dites que ce } \\
\text { qui est approprié) }\end{array}$ \\
\hline 6) Evitez les obscurités. & \multirow{4}{*}{$\begin{array}{l}\text { - Une maxime de manière } \\
\text { (soyez clair) spécifiée en quatre } \\
\text { sous-maximes : } \\
6), 7), 8 \text { ) et } 9 \text { ). }\end{array}$} & \multirow{4}{*}{$\begin{array}{l}\text { - La modalité (soyez bref et } \\
\text { précis; évitez l'obscurité et } \\
\text { l'ambiguïté) }\end{array}$} \\
\hline 7) Evitez les ambiguïtés. & & \\
\hline 8) Soyez bref. & & \\
\hline 9) Soyez ordonné. & & \\
\hline
\end{tabular}

Le principe coopératif se trouve détaillé, dans le texte européen, sous la forme de quatre critères (quantité, qualité, pertinence, modalité). C'est la notion de coopération, plutôt que celle de pertinence, qui a donc été retenue pour fédérer ces différents paramètres. Le principe maximaliste de pertinence, qui stipule que tout acte de communication communique la présomption de sa propre pertinence et explique ipso facto pourquoi le destinataire accepte de prêter attention et de traiter l'acte de communication, n'est pas véritablement identifié comme tel.

Le principe coopératif n'apparaît dans le texte que comme un item parmi d'autres. Il est mentionné sous l'en-tête "la capacité de gérer et de structurer le discours " au même titre que l'« organisation thématique ", la " cohérence et la cohésion » ou l'« organisation logique », si l'on s'en tient aux trois premiers exemples. Cela a pour effet de niveler ces différents éléments, alors qu'il s'établit au contraire des relations causales entre le principe gricéen et l'organisation textuelle et qu'il est par conséquent possible d'introduire une hiérarchisation (cf. infra).

En rappelant en préambule le principe général qui préside aux maximes conversationnelles de Grice ( faites en sorte que votre contribution corresponde à ce qui est exigé, au niveau où elle a lieu, par la finalité ou le sens acceptés de l'échange conversationnel dans lequel vous êtes engagé(s) ", les auteurs du Cecrl évoquent, sans pour autant insister sur ce point capital, la notion de finalité qui guide, dans le contexte situationnel, toute interaction verbale et, de manière plus large, toute action. 


\section{Conclusion partielle}

considérations, pourrait se définir comme l'aptitude du locuteur / scripteur à sélectionner et à arranger au mieux les éléments linguistiques, selon la visée d'effet recherché et les circonstances énonciatives dans lesquelles il se trouve. Il s'établit donc une relation causale entre d'une part, sens d'intention / contexte situationnel et d'autre part adaptation / sélection / arrangement textuel. De manière concrète, on ne s'y prendra pas de la même façon si l'on veut emprunter de l'argent à un ami ou à un banquier. De même, pour la négociation d'un contrat difficile, on recherchera la meilleure façon de présenter ce que l'on a à dire et l'on accordera un soin particulier aux axes les plus délicats de l'échange. Voilà pourquoi, si plusieurs personnes sont susceptibles d'être concernées par le projet, la direction choisira en général celle qui a les meilleures compétences pragmatiques. européen, a sans doute le mérite d'intégrer une composante majeure des théories classiques sur la pragmatique (à savoir le rôle de la situation sur la parole) à la linguistique textuelle (conçue comme l'organisation des unités linguistiques). Un locuteur organise son discours selon qu'il cherche à l'adapter au contexte ou qu'il vise à modifier celui-ci. Autrement dit, la parole peut influer sur la situation tout comme la situation peut influer sur la parole (cf. supra $\$ 1.1 .1$ \& 1.1.2.). On voit comment l'art et la manière d'agencer le matériau linguistique ressortit à la compétence pragmatique, qui peut alors faire l'objet d'un véritable apprentissage.

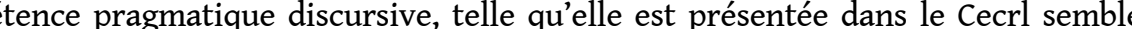
donc bien liée à une pragmatique intégrée à la sémantique, c'est-à-dire à une théorie scientifique, mais les liens entre l'ancrage officiel (didactique) et l'ancrage théorique (théorie de référence) se perdent dans l'implicite et ne peuvent être rappelés que par la mise en évidence de relations logiques et un retour à des considérations diachroniques (cf. infra conclusion).

En résumé, dans ce premier volet du Cecrl sur la compétence pragmatique, les fondements théoriques classiques sont plus ou moins difficiles à identifier. Cela ne signifie pas qu'ils n'aient pas exercé d'influence sur l'élaboration du texte européen, mais il faut bien admettre que, hormis la référence explicite aux maximes conversationnelles de Grice, ils ne s'imposent guère comme une évidence. C'est fondamentalement la linguistique textuelle, dont l'appartenance à la pragmatique est parfois controversée (cf. supra $\S 1.3$. et conclusion générale), qui constitue les fondements théoriques les plus visibles de cette première partie.

\section{La compétence fonctionnelle}

La compétence fonctionnelle correspond à la deuxième composante de la compétence pragmatique. Elle se scinde à son tour en trois éléments : les micro-fonctions, les macrofonctions et les schémas d'interaction. 


\section{Les micro-fonctions}

41 Les micro-fonctions «sont des catégories servant à définir l'utilisation fonctionnelle d'énoncés simples (généralement courts), habituellement lors d'une intervention dans une interaction » (Cecrl : 98 ). Elles font l'objet d'une classification extrêmement détaillée dans Threshold Level $1990^{5}$, chapitre 5, classification reproduite dans le Cecrl. Six microfonctions sont répertoriées. Chaque micro-fonction est déclinée à son tour en souscomposantes.

Les micro-fonctions, telles qu'elles sont évoquées dans le cadre européen (exprimer l'accord, le désaccord, l'obligation, le goût, etc.) sont de toute évidence un vestige de l'approche notionnelle-fonctionnelle des années 80, également connue sous le nom de première approche communicative (cf. note 5). L'ouvrage de Wilkins, publié en 1976, Notional Syllabuses, a contribué à fournir le support scientifique pour une intégration de l'approche communicative dans l'enseignement des langues étrangères en France. Wilkins propose un découpage de la langue fondé sur le sens et non pas sur la grammaire, ceci en vue d'identifier plus précisément des besoins de communication. Pour bâtir son analyse, il a eu recours à deux concepts théoriques, les notions et les fonctions ${ }^{6}$.

Ces fonctions me paraissent entretenir un lien étroit avec la pragmatique telle que je l'ai définie dans son deuxième sens. Par exemple, "s'excuser ", " persuader ", " obliger ", « autoriser », etc. sont à l'évidence des actes de langage (illocutoires et/ou perlocutoires) qui instaurent un type de rapport particulier entre les interlocuteurs et visent à transformer, par le discours lui-même, l'environnement où ils sont produits (cf. supra $\S$ 1.).

Enfin, l'approche notionnelle-fonctionnelle relève de la compétence pragmatique dans la mesure où elle vise à proposer à l'apprenant un choix de notions et fonctions susceptibles d'être mobilisées selon la situation. Cet objectif complète ma définition inférée précédemment à partir du texte européen (cf. supra $\$$ 3.1.2.) :

L'aptitude du locuteur / scripteur à sélectionner et à arranger au mieux les éléments linguistiques, selon la visée d'effet recherché et les circonstances énonciatives dans lesquelles il se trouve sera stimulée si l'on met à sa disposition des outils linguistiques présélectionnés (notions et fonctions), réutilisables selon la nécessité du moment.

\section{Les macro-fonctions}

La macro-fonction est ainsi définie :

Une macro-fonction se caractérise par sa structure interactionnelle. Il se peut que des situations plus complexes aient une structure interne composée de séquences de macro-fonctions qui, dans de nombreux cas, s'ordonnent selon les modèles formels ou informels de l'interaction sociale (schèmes) [...] [Ce] sont des catégories servant à définir l'utilisation fonctionnelle du discours oral ou du texte écrit qui consistent en une suite (parfois importante) de phrases (Cecrl:98 ).

Voici quelques exemples de macro-fonction: description, narration, commentaire, exposé, exégèse, explication, instruction, argumentation, démonstration, persuasion, etc.

La différenciation entre micro-fonctions et macro-fonctions me paraît globalement envisagée en termes quantitatifs: énoncés simples, courts, correspondant à une intervention, pour les micro-fonctions, suite parfois importante de phrases, pour les macro-fonctions. Pourtant, l'item «explication», logé par les auteurs dans les macro- 
fonctions pourrait, dans bien des cas, pouvoir se résumer à UNE réponse à une demande d'information. "Donner et demander des informations", est rangé, quant à lui, sans justification apparente, dans la catégorie des micro-fonctions. De même «exprimer son désaccord ", mentionné dans la catégorie des micro-fonctions ne me semble pas étranger à une stratégie d'" argumentation " ou de "démonstration ", classées, quant à elles, dans les macro-fonctions. La démarcation établie entre micro-fonctions et macro-fonctions ne s'impose donc pas, loin s'en faut, comme une évidence.

La distinction entre ces deux notions peut être rapprochée de celle opérée par Maingueneau entre macro-actes et micro-actes de langage. Elle repose là aussi sur des critères quantitatifs :

La pragmatique textuelle est confrontée à des séquences plus ou moins longues d'actes de langage qui permettent d'établir à un niveau supérieur, une valeur illocutoire globale, celle de macro-actes de langage (1991:174).

Les macro-actes désignent des unités pragmatiques de taille supérieure à la phrase (ou l'énoncé).

Dans une théorie fondée sur les actes de langage, il semble possible d'envisager la relation entre macro et micro-fonctions en termes de contenu / contenant. Ainsi, dans le schéma général pour l'achat de marchandises ou de services ( $c f$. infra), les différents actes locutoires et illocutoires produits par l'employé (saluer, renseigner, conseiller, etc.) sont tous subsumés par une intention perlocutoire qui vise à provoquer l'achat de tel ou tel produit. Sous le couvert de micro-actes se dissimule la finalité économique du macro-acte (que l'on pourrait rapprocher également de la structure pragmatique du discours publicitaire proposé par Jean-Michel Adam et Marc Bonhomme (1997 : 25).

51 Sans doute aura-t-on remarqué la relation établie ici entre actes de langage (locutoire, illocutoire, perlocutoire) et fonctions langagières (macro-fonctions, micro-fonctions). Ces deux approches de la langue ne sont pas mutuellement exclusives mais il importe de souligner que le Cecrl, contrairement au Niveau Seuil, ne recourt pas explicitement aux actes de langage tels que les décrivent Austin ou Searle. Il appréhende le contenu linguistique en termes de fonctions langagières.

\section{La compétence de conception schématique}

\section{Présentation et extraits choisis}

52 La compétence de conception schématique n'est pas analysée en tant que telle dans le cadre européen. On sait simplement qu'elle traite «de la connaissance que l'utilisateur/ apprenant a des principes selon lesquels les messages sont [...] segmentés selon des schémas interactionnels et transactionnels » (op. cit.: 96). On aurait pu s'attendre, en toute logique, que le développement consacré aux schémas d'interaction soit utilisé pour illustrer cette définition. Ce n'est pas le cas. Les schémas d'interaction sont rangés, avec les microfonctions et les macro-fonctions, dans la rubrique intitulée "La compétence fonctionnelle ». Les auteurs en donnent pourtant la définition suivante :

modèles d'interaction sociale qui sous-tendent la communication, tels que les modèles d'échanges verbaux. Les activités de communication interactive [...] comprennent des suites structurées d'actions effectuées à tour de rôle par les différentes parties. (Cecrl : 99) 
53

Autant de raisons, donc, pour réserver aux interactions un traitement à part et les considérer, en définitive, comme une illustration de la troisième compétence pragmatique, la compétence de conception schématique.

Le Cecrl reproduit le schéma général proposé au chapitre 8 dans le Threshold Level (1990 85-86), pour décrire les interactions lors de l'achat de marchandises ou de services. Cinq étapes sont ainsi proposées :

1. Se rendre à l'endroit de la transaction

2. Établir le contact

3. Choisir la marchandise/le service

4. Échanger les marchandises contre un paiement

5. Prendre congé

Chaque étape est à son tour finement détaillée, par exemple, l'étape 3, «choisir la marchandise/le service » :

3.1 Identifier la catégorie de marchandises/services désirée

3.1.1 Rechercher l'information

3.1.2 Donner l'information

3.2 Identifier les choix

3.3 Discuter le pour et le contre des différentes possibilités (par exemple, la qualité,

le prix, la couleur, la dimension des marchandises)

3.3.1 Rechercher les informations

3.3.2 Donner les informations

3.3.3 Demander conseil

3.3.4 Conseiller

3.3.5 Demander les préférences

3.3.6 Exprimer ses préférences, etc.

3.4 Identifier les marchandises choisies

3.5 Examiner les marchandises

3.6 Donner son accord sur l'achat

ou l'étape 5 , "prendre congé »:

5.1 Exprimer sa satisfaction (mutuelle)

5.1.1 L'employé exprime sa satisfaction

5.1.2 Le client exprime sa satisfaction

5.2 Échanger des menus propos (par exemple sur le temps, les potins)

5.3 Échanger des salutations finales

5.3.1 Salutations de l'employé

5.3.2 Salutations du client

\section{Un schéma plus ou moins schématique}

On voit bien que cet exemple d'interactions n'est pas la transcription d'un dialogue authentique mais, comme le précisent clairement les auteurs, un "schéma général » ou encore un "modèle ", constitué, en l'occurrence d'une suite d'étiquettes métalangagières, chronologiquement ordonnées ${ }^{7}$. Les différents actes de paroles sont ainsi référés sous une forme essentiellement nominale ou quasi-nominale (nom ou infinitif e.g. "salutation de l'employé » / "saluer l'employé»), plus rarement sous une forme verbale conjuguée (« l'employé exprime sa satisfaction»). Dans tous les cas, et ce malgré la situation d'interlocution visée, ce sont des personnes de troisième rang (nom, infinitif, délocuté « il ») qui sont systématiquement utilisées.

57 L'interaction verbale prend place dans un environnement référentiel précis mais potentiellement ouvert, présenté dans le Cecrl à l'aide d'un paradigme d'exemples relevant d'un même champ isotopique ("boutique, magasin, restaurant, hôtel », etc.). Il 
s'agit en effet de lieux dont les propriétés sociales imposent un "cadrage de l'expérience " (Goffman, 1991), générateur d'attentes spécifiques ou, pour reprendre l'expression d'E. Roulet (1999: 52-55), de lieux qui renvoient tous à un «cadre actionnel» permettant de décrire la " position actionnelle » de chaque interactant : statuts sociaux (libraire et client), rôle praxéologique dans l'interaction (prestataire et demandeur de service), sensibilité plus ou moins grande aux problèmes de "face" (image positive ou négative que l'on donne de soi) ou encore de " place » (relation de domination entre les interactants).

Si l'on admet que la structure hiérarchique d'un dialogue est le produit de processus de négociation sous-jacents, la section 3, "Choisir la marchandise / le service », présente six négociations successives correspondant à six échanges, un échange étant fait de " constituants d'un rang égal ou supérieur à l'intervention » (Roulet \& al., 2001 : 54). La première négociation ("rechercher l'information») est suivie d'une réaction positive («donner l'information »). Il est bien évident, que dans un dialogue authentique, la réaction peut s'avérer positive, négative mais aussi mitigée, imprécise ou laconique, pouvant ainsi donner lieu à une nouvelle négociation, susceptible alors d'aboutir à une ratification. Ces différentes bifurcations, caractéristiques d'un échange situé, relèvent là encore de " configurations émergentes ", absentes donc d'un modèle dialogique qui cherche à mettre en évidence des invariants.

Il convient en effet de souligner que le schéma présenté dans le cadre ne sélectionne que les principales étapes attendues dans ce type d'environnement dialogique: pratiques rituelles (ouverture / clôture), négociations transactionnelles (achat / vente; renseignement), etc. Il ne vise pas l'exhaustivité. Autrement dit, il ne mobilise pas toutes les potentialités susceptibles d'être actualisées en contexte spécifique. Il met en avant ce que Fillietaz (2002) appelle des « ressources schématiques de l'agir ». Dans des «incursions» situées, seules certaines des potentialités mises en évidence par un schéma général, tel que celui proposé pour l'achat de marchandises ou de services, seront effectivement actualisées. Fillietaz pratique à cet égard une différenciation pertinente entre " activités ", relevant d'une représentation praxéologique schématique (cf. supra § 1.3.) et "actions ", relevant de structures praxéologiques émergentes, c'est-à-dire correspondant à des réalités praxéologiques effectivement négociées par des acteurs bien déterminés évoluant dans des situations particulières. incursion en librairie (E. Roulet, 1995 : 132) :

Figure 2 : Représentation praxéologique

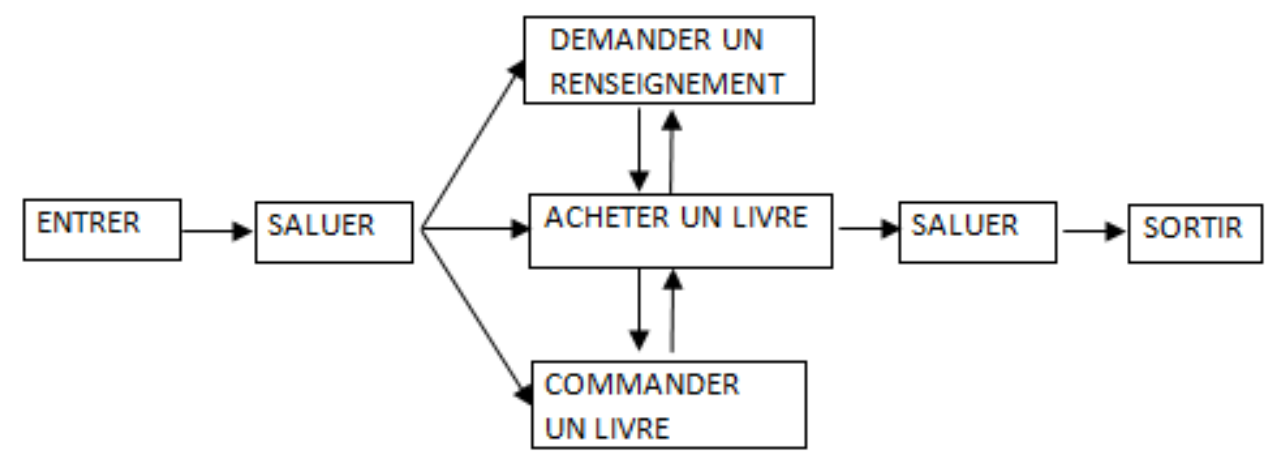


61 Cet organigramme, conçu à partir d'un dialogue effectif, ne prend en compte que l'interaction en magasin. Il occulte notamment l'étape 1 mentionnée dans le Cecrl («se rendre à l'endroit de la transaction »), étape pourtant motivée par une même démarche téléologique. La représentation praxéologique de Roulet offre cependant l'avantage de mettre au centre l'objet même de la transaction ("acheter un livre ») autour duquel s'organisent les différents actes qui structurent l'interaction. Elle présente enfin, en l'occurrence, une configuration plus épurée que celle qui apparaît dans le cadre. On peut être même tenté de considérer que le schéma du cadre européen s'apparente plus à un script ou un scénario qu'à une représentation praxéologique telle qu'on la trouve dans les analyses modulaires du discours. Fillietaz attire l'attention sur la nécessité de bien distinguer les deux formes : un script (ou un scénario) exerce, selon lui, un déterminisme plus fort sur le déroulement des actions effectives que l'on pourrait mettre en scène à partir d'un tel canevas, une représentation praxéologique n'explicite «que des attentes issues d'expériences passées intersubjectivement partagées » (Roulet \& al, . $2001: 110$ ).

La dichotomie opérée par Fillietaz pose le problème d'une distinction claire entre les différentes formes de représentation de l'interaction: où situer la juste frontière entre représentation généralisante et représentation particularisante, intégrant des configurations émergentes? Il me paraît difficile de déterminer un seuil critique en la matière ${ }^{8}$.

Je noterai simplement que des paramètres tels que les notions de «position » ou de «place ", se laissent difficilement réduire à une représentation schématique mais qu'ils pourront en revanche figurer dans un scénario détaillé. Je soulignerai également que les modalités suprasegmentales, mais aussi non verbales (kinésiques, proxémiques), n'apparaissent ni dans le schéma général du Cecrl ni dans la représentation praxéologique de Roulet. Elles pourraient pourtant être intégrées à une matrice interactionnelle et donner lieu à un travail de systématisation. Dans les deux cas, elles font l'objet d'un traitement à part.

Il me paraît tout au plus possible d'appréhender la question en termes de gradient ou de continuum faisant insensiblement passer d'une "représentation schématique ", qui tend vers le virtuel, le général, le large à une « configuration émergente » qui tend vers l'actuel, le particulier, l'étroit. En schéma :

Figure 3 : Représentations schématique et convergente appréhendées sous la forme d'un continuum

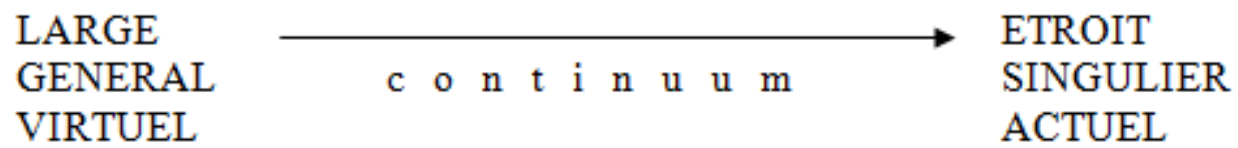

65 Il devient alors possible de prélever différentes saisies sur cet axe qui mène du large à l'étroit et produire ainsi toutes sortes de schémas, plus ou moins complexes, plus ou moins détaillés, plus ou moins directifs. L'opération inverse, celle qui mène de l'étroit au large, est également envisageable. Cette démarche, de type sémasiologique, se fonde sur des interactions effectives pour mettre en évidence les invariants de leur structuration.

Une activité envisageable consisterait à faire produire un dialogue sur un même thème à partir d'une représentation praxéologique telle que la conçoit Roulet ou d'un schéma général tel qu'il est proposé dans le Cecrl. Pour des raisons aisément compréhensibles, il y a fort à parier, que les divergences entre produits finis soient plus importantes si elles 
sont issues d'un modèle universalisant que si elles sont dérivées d'un modèle particularisant. Il est également peu probable qu'une représentation, aussi détaillée soient-elle, donne lieu à des productions de dialogues rigoureusement identiques. L'intérêt d'une représentation praxéologique est de conserver une valeur typifiante.

La démarche méthodologique inverse, adoptée par les analystes du discours, consiste à partir d'interactions situées pour en abstraire des représentations praxéologiques. Selon leur degré de précision, en particulier la mise en évidence de macro- ou de microstructures, elles donneront lieu à des schémas plus ou moins sophistiqués. Dans le cadre de l'apprentissage, une activité possible consisterait à faire dégager des invariants à partir de dialogues authentiques, la démarche de l'apprenant rejoignant alors celle du chercheur.

\section{Conclusion}

Quels sont donc les liens que l'on peut tisser entre pragmatique et compétence pragmatique dans le texte européen? Est-il possible de dériver l'une de l'autre? La compétence pragmatique, telle qu'elle est décrite dans le Cecrl, s'articule autour de trois composantes, la compétence discursive, la compétence fonctionnelle et la compétence de conception schématique. La première met l'accent sur la cohésion et la cohérence du texte oral ou écrit, la seconde sur les fonctions langagières, la troisième sur la structure interactionnelle ou praxéologique du dialogue. En simplifiant un peu, on considérera que le texte européen s'inspire dans le premier cas de la linguistique textuelle, dans le second, de l'approche notionnelle-fonctionnelle des années 80 , dans le troisième, de l'analyse du discours, la délimitation entre linguistique textuelle et analyse du discours demeurant sujette à controverses.

Quelle est la part de l'héritage scientifique privilégiée par le Cadre Européen dans son traitement de la compétence pragmatique ? Force est de constater la prépondérance du rôle joué par l'analyse textuelle et l'analyse du discours. Mais le problème se pose de savoir si ces domaines font partie ou non de la pragmatique. Avons-nous affaire à des champs d'étude distincts ou imbriqués? La question est loin d'être résolue. Il semble malgré tout possible de déceler des zones de recouvrement. Par exemple, nombreux sont les linguistes, notamment dans les pays anglo-saxons, qui assimilent analyse du discours et analyse conversationnelle, celle-ci constituant le champ d'étude privilégié des courants interactionnistes, lesquels sont étroitement liés aux conceptions pragmatiques. Le lien entre pragmatique et analyse du discours ne s'établit, on le voit, que de façon médiate.

En revanche, les théories plus anciennes ne suscitent guère de débats quant à leur appartenance à la pragmatique. Mais, contrairement au cas de figure précédent, les emprunts pratiqués par le Cecrl sont plus délicats à mettre en évidence, peut-être parce qu'ils sont relégués dans l'implicite, à quelques rares exceptions près, comme les maximes conversationnelles de Grice, auxquelles il est fait explicitement référence. Cela ne veut pas dire que le Cecrl fasse abstraction de cet arrière-plan scientifique, mais ce n'est qu'au prix d'une analyse minutieuse que l'on parvient à mettre à jour l'apport théorique sous-jacent.

71 Certes, définir la compétence pragmatique à la lumière d'un héritage scientifique ne constituait pas le but visé par les auteurs du texte européen. Que l'assise théorique transparaisse de façon explicite ou implicite, le cadre s'impose en effet comme un texte 
auto-suffisant, "refermé sur lui-même dans une douce autarcie » (Chevallard, 1991:22), qui anesthésie la perception d'un savoir savant ayant pu, de près ou de loin, jouer un rôle dans son élaboration. Le but de cette analyse était précisément de raviver cette perception, mettre ainsi en perspective le "savoir à enseigner ", hiérarchiser des priorités souvent nivelées dans le cadre, favoriser une conceptualisation plus fine des notions abordées, aiguiser l'esprit critique et la curiosité, bref, générer une prise de conscience de l'ancrage théorique susceptible d'aider l'utilisateur du texte européen à mieux cerner les enjeux de l'ancrage officiel.

\section{BIBLIOGRAPHIE}

Adam, J.M. \& Bonhomme, M. (1997). L'Argumentation publicitaire. Rhétorique de l'éloge et de la persuasion. Paris: Nathan Université.

Austin, J.L. (1962). How to do things with words. Oxford: Oxford University Press.

Austin, J.L. (1970). Quand dire c'est faire. Coll. Essais. Paris : Seuil.

Charaudeau, P. \& Maingueneau, D. (2002). Dictionnaire d'analyse du discours. Paris : Le Seuil.

Charolles, M. (1988). « Les études sur la cohérence et la connexité textuelles depuis la fin des années 1960 ». Modèles linguistiques, vol. 10, fasc.2, n²0. pp. 45-66.

Chevallard, Y. (1991). La transposition didactique, du savoir savant au savoir enseigné. Grenoble : La Pensée Sauvage, Editions.

Conseil de l'Europe, (2001). Cadre européen commun de référence pour les langues, Apprendre, enseigner, évaluer. Strasbourg : Didier.

Deyrich, M.C. (2001). « Quelles médiations pour une gestion de la transposition didactique en anglais de spécialité ? » ASp 31-33. Disponible en ligne. http://asp.revues.org/1909.

Ducrot, O. \& Schaeffer, J.M. ([1972]1995). Nouveau dictionnaire des sciences du langage. Paris : Seuil.

Filliettaz, L. ( 2002). La parole en action. Eléments de pragmatique psycho-sociale. Berne: Editions NotaBene.

Gazdar, G. (1979). Pragmatics. Implicature, Presupposition, and Logical Form. NewYork : Academic Press.

Goffman, E. ([1974] 1991). Les Cadres de l'expérience. Traduit de l'anglais par Isaac Joseph. Collection « Le sens commun ». Paris : Les Editions de Minuit.

Greimas, A.J. \& Courtés, J. ([1979] 1994). Dictionnaire raisonné de la théorie du langage. Paris: Hachette.

Grice, H.P. (1975). « Logic and Conversation ». In Cole P. \& Morgan J.L. (dir.). Syntax and Semantics 3: Speech Acts. New York: Academic Press. pp. 41-58.

Halliday, M.A.K. \& Hasan, R. (1976). Cohesion in English. London: Longman.

Halliday, M.A.K. (1985). An Introduction to Functional Grammar. London: Arnold. 
Halliday, M.A.K. (2009). Language and Society, vol. 10. London : Webster.

Jakobson, R. (1963). Essais de linguistique générale. Paris : Minuit.

Levinson, S.C. (1983). Pragmatics. Cambridge: Cambridge University Press.

Lombard, P. (2008). « Remarques sur la « théorie » de la transposition didactique ». Irem de Lorraine. http://www.irem.univ-lorraine.fr/Lomb/transposition.pdf.

Maingueneau, D. (1991). L'Analyse du discours - Introduction aux lectures de l'archive. Paris : Hachette supérieur.

Maingueneau, D. (1996a). Aborder la linguistique. Paris : Seuil.

Maingueneau, D. (1996b). Les termes clés de l'analyse du discours. Paris : Seuil.

Moeschler J. (2001). «Pragmatique : état de l'art et perspectives ». Marges linguistiques, $\mathrm{n}^{\circ} 1$.

http://www.marges-linguistiques.com

Moeschler, R.J. \& Reboul A. (1994). Dictionnaire Encyclopédique de Pragmatique. Paris : Éditions du Seuil.

Morris, C.W. (1939). The Foundations of the Theory of Signs, vol. I, no 2. Chicago: International Encyclopedia of Unified Science.

Peirce C. S. (1931-1935). Collected Papers. Cambridge: Harvard University Press.

Quivy, M. \& Tardieu, C. ([1997] 2002). Glossaire de didactique de l'anglais. Paris : Ellipses.

Roulet, E, Fillietaz, L \& Grobet, A. (1985). L'articulation du discours en français contemporain. Berne : Peter Lang.

Roulet, E., Filliettaz, L., Grobet, A. \& Burger, M. (2001). Un modèle et un instrument d'analyse de l'organisation du discours. Berne : Peter Lang.

Salles, M. (2006). « Cohésion-cohérence : accords et désaccords ». Corela, Hors-série n 5, http:// corela.revues.org/1426.

Schaeffer, J.M. ([1999] 2008). Pourquoi la fiction. Paris : Seuil.

Searle, J.R. (1972). Les Actes de langage. Paris : Hermann.

Sperber D. \& Wilson D. (1986). Relevance. Communication and Cognition. Oxford: Blackwell.

Sperber, D. \& Wilson, D. (1989). La pertinence. Communication et cognition. Paris: Minuit.

Van Eck, J.A (1980). Threshold Level English. Londres: Pergamon Press.

Van Eck, J.A. \& Trim, J.L.M. (1990). Threshold Level 1990. Strasbourg : Conseil de l'Europe.

Wilkins D.A., (1976). Notional syllabuses. Oxford : University Press, Oxford.

\section{NOTES}

1. Morris distinguait trois domaines dans l'appréhension du langage : 1) la syntaxe qui concerne les rapports entre les signes dans l'énoncé complexe ; 2) la sémantique, qui traite des rapports entre les signes et la réalité ; 3) la pragmatique qui s'intéresse aux rapports entre les signes et leurs utilisateurs.

2. On parle aussi d' « indexicaux» (et, par dérivation, d' "indexicalisation » et d'« indexicalité »).

3. Expression empruntée à Chevallard, qui en fait le titre de son ouvrage de 1991 (lui-même une reprise de l'ouvrage de 1985), titre assorti du sous-titre du savoir savant au savoir enseigné. L'auteur 
considère que le savoir enseigné est issu d'un savoir savant qui le fonde culturellement et scientifiquement. La transposition didactique concerne l'adaptation du savoir savant au savoir enseigné et l'écart susceptible de se creuser entre les deux lors de cette transposition. Le texte européen ne saurait toutefois être assimilé à une discipline enseignée au même titre que les mathématiques sur lesquelles l'auteur fonde en l'occurrence l'essentiel de son analyse ou l'anglais de spécialité (ASP), abordé par Deyrich (2001) sous l'angle de la médiation didactique, elle-même issue des principes fondateurs élaborés par Chevallard. La notion de transposition didactique ne s'applique donc que partiellement au cadre européen et ne se justifie ici que par la portée délibérément simplificatrice du texte européen. Sur un plan plus général le principe même d'une " théorie de la transposition didactique » en tant que science à part entière a été fortement remis en cause (Lombard 2008).

4. Voir Cecrl, § 5.2.3.1, p. 96.

5. Le Threshold Level ou, en français, le Niveau seuil (Van Eck 1975 et Van Eck \& Trim, 1990), a fortement influencé en France les approches communicatives de première génération (années 80 ), également connue sous le nom d'«approche notionnelle-fonctionnelle " et de $2^{\text {ème }}$ génération (années 90). Il s'agit toujours, dans le deuxième cas, d'une approche notionnellefonctionnelle mais celle-ci intègre également les courants cognitiviste et constructiviste. Dans la première et la deuxième édition du Threshold Level, l'accent est mis sur la langue telle qu'elle est utilisée pour répondre aux besoins quotidiens d'un adulte vivant dans un pays étranger, sur la langue comme outil social (utilisation de la langue pour faire ce qu'il est convenable de faire dans la situation interlocutive) et sur l'interaction. L'approche notionnelle-fonctionnelle met donc en avant le rôle joué par la situation sur la mobilisation des moyens langagiers appropriés (dimension pragmatique), ainsi que l'adaptation du niveau de langue à la situation (dimension socio-linguistique).

6. On pourrait définir succinctement la notion comme l'objet du discours (ce de quoi l'on parle : temps, fréquence, etc.) et la fonction comme son objectif (ce pour quoi l'on parle: apport d'information, ordre, conseil, etc.) (Quivy \& Tardieu, 2002 : 169).

La notion se présente sous une forme nominale (espace, durée, couleurs, la localisation, etc.), les fonctions sous une forme verbale (speech act) (se présenter, parler de soi, parler des autres, etc.).

7. Le schéma général de l'achat de marchandises ou de services n'est en réalité dicté que par des contraintes de successivité expérientielle. Ce critère de structuration, bien que fondamental, n'est pas explicitement identifié.

8. Il n'est pas inconcevable, a priori, de convertir le schéma général du Cecrl en une représentation plus élaborée mais de même type que celle proposée dans la figure 1. Cela ne modifie en rien la question générale de savoir comment évaluer le degré de sophistication que peut supporter une telle représentation sans nuire pour autant à la clarté de son interprétation.

\section{RÉSUMÉS}

Cette étude se penche sur la façon dont la compétence pragmatique est abordée dans le Cadre Européen Commun de Référence pour les langues (Cecrl). Elle vise plus précisément à cerner le rôle que les différents courants qui ont jalonné l'histoire de la pragmatique ont pu exercer dans l'élaboration de cette compétence. Définir la compétence pragmatique à la lumière d'un héritage scientifique ne constituait pas le but visé par les auteurs du texte européen. Que l'assise théorique transparaisse de façon explicite ou implicite, le Cadre Européen s'impose en effet 
comme un texte auto-suffisant. Il n'est toutefois par illégitime de penser qu'une prise de conscience de l'ancrage théorique sous-jacent puisse aider l'utilisateur du Cadre à mieux cerner les enjeux de l'ancrage didactique officiel.

This paper focuses on the way pragmatic competence is handled in the Common European Framework for Languages (CEF). It is more precisely intended to help the reader understand how the major trends marking out the history of pragmatics influenced the development of that competence. Defining pragmatic competences in the light of their scientific legacy was in no way what the authors of the European Framework actually had in mind. The CEF appears as a selfsufficient text, no matter how explicitly or implicitly the theoretical background may be resorted to. Yet it is not totally illegitimate to consider that an enhanced grasp and awareness of the underlying scientific approach makes for better understanding of the didactic approach.

\section{INDEX}

Mots-clés : pragmatique, compétence pragmatique, acte de parole, analyse du discours, représentation praxéologique

Keywords : pragmatics, pragmatic competence, speech act, discourse analysis, praxeologic representation

\section{AUTEUR}

\section{YVES BARDIÈRE}

EA 609 LIDILEM (Laboratoire de linguistique et didactique des langues étrangères et maternelles), Université Stendhal-Grenoble 3.

Yves Bardière est agrégé d'anglais et professeur des universités à Stendhal-Grenoble 3. Ses recherches portent plus particulièrement sur la linguistique contrastive mais aussi la didactique des langues, la didactique professionnelle et l'étude de l'anglais en tant que langue de spécialité (domaine naval, aéronaval et OTAN).

Courriel : y.bardiere[at]laposte.net 